OPEN ACCESS

Edited by:

Tamer Demiralp,

Istanbul University, Turkey

Reviewed by:

Ahmet Omurtag,

Nottingham Trent University,

United Kingdom

Pietro Aricò,

Sapienza University of Rome, Italy

*Correspondence:

Kobi Gal

kobig@bgu.ac.ll

Oren Shriki

shrikio@bgu.ac.il

Received: 30 December 2018 Accepted: 22 May 2019

Published: 11 June 2019

Citation:

Friedman N, Fekete T, Gal K and Shrik

O (2019) EEG-Based Prediction of

Cognitive Load in Intelligence Tests.

Front. Hum. Neurosci. 13:191.

doi: 10.3389/fnhum.2019.00191

\section{EEG-Based Prediction of Cognitive Load in Intelligence Tests}

\author{
Nir Friedman ${ }^{1,2}$, Tomer Fekete ${ }^{2}$, Kobi Gal ${ }^{1,3 *}$ and Oren Shriki ${ }^{2,4,5 *}$ \\ ${ }^{1}$ Department of Software and Information Systems Engineering, Ben-Gurion University of the Negev, Beersheba, Israel, \\ ${ }^{2}$ Department of Cognitive and Brain Sciences, Ben-Gurion University of the Negev, Beersheba, Israel, ${ }^{3}$ School of Informatics, \\ University of Edinburgh, Edinburgh, United Kingdom, ${ }^{4}$ Department of Computer Science, Ben-Gurion University of the \\ Negev, Beersheba, Israel, ${ }^{5}$ Zlotowski Center for Neuroscience, Ben-Gurion University of the Negev, Beersheba, Israel
}

Measuring and assessing the cognitive load associated with different tasks is crucial for many applications, from the design of instructional materials to monitoring the mental well-being of aircraft pilots. The goal of this paper is to utilize EEG to infer the cognitive workload of subjects during intelligence tests. We chose the well established advanced progressive matrices test, an ideal framework because it presents problems at increasing levels of difficulty and has been rigorously validated in past experiments. We train classic machine learning models using basic EEG measures as well as measures of network connectivity and signal complexity. Our findings demonstrate that cognitive load can be well predicted using these features, even for a low number of channels. We show that by creating an individually tuned neural network for each subject, we can improve prediction compared to a generic model and that such models are robust to decreasing the number of available channels as well.

Keywords: brain-computer interface, electroencephalography, cognitive load, machine learning, Raven's matrices

\section{INTRODUCTION}

The performance of complex tasks requires the integration of various mental resources, such as task-related knowledge, working memory, attention and decision making. However, our brains have limited resources for processing and integrating information. The concept of cognitive load generally refers to the relative load on these limited resources (Sweller et al., 1998; Coyne et al., 2009).

Cognitive workload has been explored from different perspectives. Brouwer et al. (2012) refer to workload as the working memory load in an n-back task. Mills et al. (2017) use simple truefalse questions for eliciting low workload and open-ended questions, which require more precise memory, for eliciting high workload. Other studies have emphasized the role of skill acquisition in modeling cognitive load (Sweller et al., 1998). Logan (1985) show that when subjects acquire a skill and learn how to perform a task in an automatic manner, their cognitive workload decreases (Borghini et al., 2017). Thus, the cognitive load depends not only on task complexity but also on the subject's skill at the given task. A highly complex task performed by a non-skilled individual would result in high cognitive load, whereas a simple task performed by a skilled individual would result in low cognitive load. For example, Stevens et al. (2006) assessed subjects as they were learning to diagnose disorders of organ systems and Mak et al. (2013) focused on performance improvement in a visual-motor task. Both studies showed a decrease in cognitive load metrics, with an increase in task familiarity. In all of these studies, the relative difficulty of the task is seen as a proxy for its associated cognitive load. The difficulty was assessed using a variety of approaches, such as the 
type of questions (true-false vs. open ended), subject performance and even participant subjective ratings. A major limitation of many studies is that the levels of difficulty were not rigorously defined. Here, we chose a setting in which problem difficulty was rigorously validated and is commonly used in the psychological literature (see below). Another limitation of previous studies is that cognitive workload was assessed using discrete levels, often only two or three levels (Aricò et al., 2016a,b). In the present study, we use a continuous scale for workload.

In addition to behavioral measures, there is a growing interest in assessing cognitive workload using physiological measures, such as pupil diameter (Palinko et al., 2010). The focus of this paper is on quantifying cognitive workload using measures based on electroencephalography (EEG). Several studies have previously developed EEG-based measures for cognitive load. In particular, it was found that the ratio between the theta power (4$8 \mathrm{~Hz})$ and the alpha power $(8-12 \mathrm{~Hz})$, as well as the ratio between the beta power $(12-30 \mathrm{~Hz})$ and the alpha power and several related combinations, provided informative indices concerning task engagement and cognitive workload (Pope et al., 1995; Stevens et al., 2006; Mills et al., 2017). Other researchers came to similar conclusions, namely that the relation between different spectral features can help predict cognitive load from EEG (Gerě and Jaušcvec, 1999; McDonald and Soussou, 2011; Conrad and Bliemel, 2016). This study aimed to further expand these studies and develop continuous and more accurate EEG-based measures of cognitive load. Furthermore, we tried to examine the utility of additional measures, in particular network connectivity and signal complexity.

We focused on recording EEG during performance of a wellknown psychological assessment tool, the advanced progressive matrices test (Raven, 2000), which is commonly used to measure general intelligence. The test is composed of different problems that involve the manipulation of shapes. Problems are presented to subjects at increasing levels of difficulty. The difficulty of each problem is validated across a large number of subjects (Forbes, 1964; Arthur et al., 1999), in the sense that more difficult questions lead to a higher error rate in the population.

Here, we adopt problem difficulty as the operational definition of cognitive load and demonstrate that it can be predicted from the subject's EEG readings. Specifically, we employ a variety of EEG measures as input to machine-learning algorithms and train them to predict problem difficulty.

As mentioned above, previous studies of EEG-based measures of cognitive load were limited in several ways. In particular, they relied mostly on spectral features and produced a simple discrete measure of either low or high load. In contrast, this paper models cognitive load in a continuous manner. In addition, we go beyond basic spectral features and examine how measures of network connectivity and signal complexity affect the prediction of cognitive load. To measure network connectivity, we used complex network analysis (CNA), which provides measures to examine functional connectivity in the brain (Bullmore and Sporns, 2009; Fekete et al., 2014). Features of neural complexity are often computed using measures of entropy, reflecting the proportion of ordered patterns that can be detected in a signal (Bullmore et al., 2009). To measure neural complexity, we focused on Lempel-Ziv (Tononi and Edelman, 1998) complexity, Multi Scale Entropy (MSE) (Abásolo et al., 2006) and Detrended Fluctuation Analysis (DFA) (Rubin et al., 2013).

The results of this paper demonstrate the applicability of using EEG and machine learning for quantifying cognitive load in wellvalidated problem-solving tasks. In particular, as EEG and other measures of brain activity become more pervasive, quantitative cognitive load measures could be used to facilitate the design of domains involving real-time problem-solving, such as e-learning, psychometric exams, military training, and more (Ikehara and Crosby, 2005; Mills et al., 2017).

\section{METHODS}

We recorded EEG from subjects while they solved the Advanced Progressive Matrices set II (Raven test). The 36 problems in the test were presented in increasing levels of difficulty. The raw EEG data were then passed through an artifact removal pipeline (see details below) before extracting EEG-based measures of spectral activity, neural complexity and network connectivity. These measures served as input to machine learning algorithms, which were trained to predict problem difficulty.

\subsection{Participants}

Fifty-two subjects (26 female and 26 male; age range 21-28, Mean $=24.55$ years, $S D=1.76$ years) participated voluntarily in the experiment, provided written informed consent and received compensation for participating. The experiment was approved by the Ben-Gurion University ethics committee. All subjects reported that they are right-handed, have normal or corrected vision, and that they have never completed any sort of intelligence test in the past. Four participants were excluded from the study because they required $10 \mathrm{~min}$ or less to solve the entire test or answered correctly 16 problems or less. An additional participant was excluded due to a compromised recording (several electrodes did not record any signal throughout the entire session).

\subsection{Experimental Paradigm}

Subjects performed the Raven's APM Set II problems (36 items in increasing difficulty level), and instructions were delivered before the test started (see Figure 1 for an example problem). The test was run with no time limit, with all the key requirements and administration instructions carefully following the manual (Raven et al., 1998). Subjects sat in a comfortable chair facing a computer screen $60 \mathrm{~cm}$ away. The test was conducted by displaying the problems on the computer screen $\left(23^{\prime \prime}, 1,920 \times 1,080\right.$ resolution, with a $2.3^{\circ}$ visual angle between each answer's corners), where the subjects were required to press a keyboard key (with their right hand) in accordance with their chosen answer number. The experiment was programmed in MATLAB ( www.mathworks.com, version 2015), using the Psychophysics Toolbox extensions (Brainard and Vision, 1997; Pelli, 1997; Kleiner et al., 2007). Each trial lasted from the presentation of the corresponding problem until subject response, and thus trial duration was variable.

EEG was recorded through the whole session using the g.Tec HIamp system (g.Tec, Austria) with 64 gel-based electrodes 


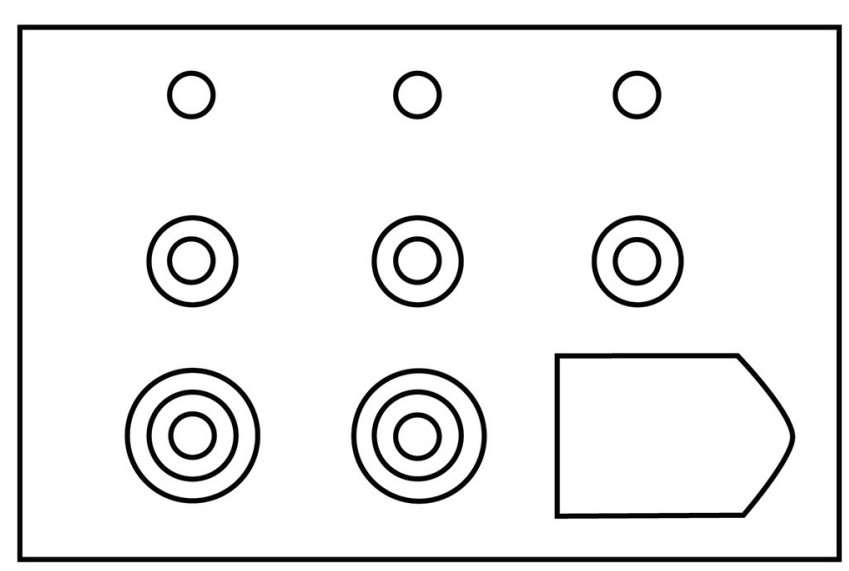

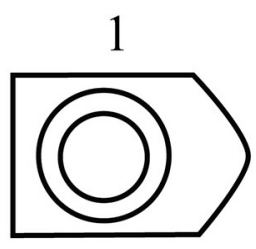

5

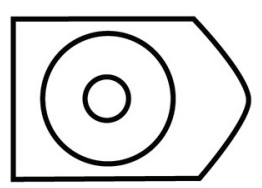

2

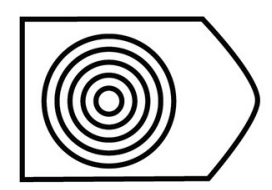

6

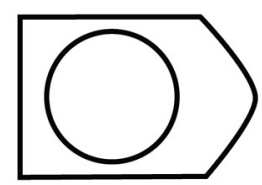

3

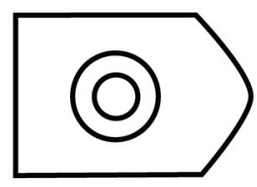

7

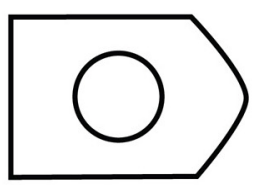

4

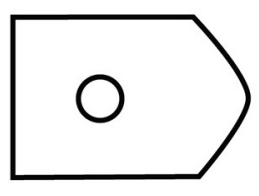

8

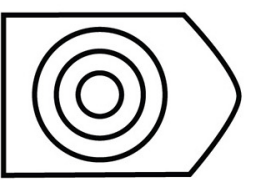

FIGURE 1 | Illustration of Raven's set II Example Problem. The subject is asked to choose the missing shape from the 8 possible options. The correct answer here is option 8.

(AgCl electrolyte gel). Electrodes were positioned according to the standard 10/20 system with linked ears reference. An impedance test and adjustment were carried out at the beginning of the session, and impedances of all electrodes were kept below $5 \mathrm{k} \Omega$. The signal was sampled at $256 \mathrm{~Hz}$ with a high-pass filter of $1 \mathrm{~Hz}$. The data were recorded using Matlab Simulink g.Tec plug-ins.

\subsection{Feature Extraction}

Data were analyzed using a combination of the EEGLAB Matlab toolbox (Delorme and Makeig, 2004) routines and custom code. Data were first high-pass filtered (cut-off $1 \mathrm{~Hz}$ ), then a customized adaptive filter was applied to suppress line-noise. This was followed by Artifact Subspace Reconstruction (Mullen et al., 2015), re-referencing to the mean, and low-pass filtering (cutoff $60 \mathrm{~Hz}$ ). Next, Infomax ICA was carried out (Bell and Sejnowski, 1995). The resulting ICs were evaluated automatically for artifacts by combining spatial, spectral and temporal analysis of ICs. ICs identified as containing ocular, muscular or cardiac artifacts were removed from data.

Various features were extracted from the EEG data:

- Power spectrum metrics (PS) - The power in 5 frequency bands (delta $[1-4 \mathrm{~Hz}]$, theta [4-8 Hz], alpha [8-12 Hz], beta
[12-30 Hz], and gamma [30-50 Hz]) was calculated for each channel across the whole trial duration. This resulted in 310 features ( 62 channels $\times 5$ bands) for each trial.

- Neural complexity metrics- We focused on three measures of complexity, specifically, Lempel-Ziv complexity (LZC) (Zhang et al., 2001), Multi Scale Entropy (MSE) (Abásolo et al., 2006) and Detrended Fluctuation Analysis (DFA) (Peng et al., 1995; Rubin et al., 2013). The LZC measure was computed as the mean of the measure across all channels, resulting in a single feature for each trial. In comparison, the MSE and DFA measures were first computed for each individual channel, and for DFA we also computed the metric for each frequency band (as described above), and a broadband [1-50Hz]. We then computed the mean, variance, maximum, minimum, $\frac{\text { mean }}{\text { variance }}$ and $\frac{\text { maximum }}{\text { minimum }}$, resulting in 6 features for the MSE, and 36 features for DFA [ 6 measures $\times(5$ bands +1 broadband $)$, resulting in 43 complexity features for each trial. Because these metrics are affected by trial duration, we calculated them for the last 2,500 samples $(\approx 10 \mathrm{~s})$ of each trial.

- Connectivity metrics - These features are based on a graph reflecting the connectivity of the underlying network. The graph comprises 62 vertices (channels); edges in the graph represent correlations between channels (there are no self 
edges). There are two approaches regarding the weight of each edge. One is to take the absolute value of the correlation as the weight of each edge. Another is to give the same weight to all edges that were kept after the thresholding process described below. We kept only the top $\mathrm{x} \%$ (we tried several thresholds) of the edges with the highest values, for example 5\% (which was what we ultimately used), meaning that we were left with 190 edges out of the $62^{2}$ (minus the 62 self edges). The graph was used to extract graph-theoretical features such as average shortest distance between nodes, small-worldness, etc. (Bullmore and Sporns, 2009). We ultimately used the mean and standard deviation of the small-worldness measure and its components, across the different thresholds.

- Basic - Simple demographic features of subjects' age and sex were used. In addition, the time it took to answer each problem was used as a feature. These features were added to all the above feature groups in the prediction phase.

\section{RESULTS}

After removal of subjects who did not meet the inclusion criteria (see Methods), we were left with 47 subjects for the analysis (24 female and 23 male; age range $21-28$, Mean $=24.55$ years, $S D=$ 1.79 years). Our goal was to estimate the cognitive workload of subjects as they were trying to solve each problem during the test.

To this end, we assumed that the difficulty level increased with every problem, as validated in previous studies (Forbes, 1964; Arthur et al., 1999). Figure 2 shows the rate of incorrect responses over all problems in our data, reflecting the established relationship between problem number and difficulty level. Interestingly, problems 24 and 29 deviated significantly from the trend (more than 3 standard deviations). For this reason, both problems were also excluded from our analysis. In addition, we only considered trials where subjects answered correctly. This is based on the premise that the cognitive load exhibited by participants for incorrect answers may not reflect the true level of the question. After excluding the subjects (180 trials) and specific problems (94 trials), as stated above, and the incorrect trials (366 trials), we were left with 1,232 trials.

For each of the 1232 correct trials, we computed different features (as detailed in the Methods) and assigned them with the corresponding difficulty level (a number between 1 and 36) as the target value. Several types of machine learning algorithms were tested in order to predict cognitive load - "Random Forest" (RF) from the sklearn python package (Buitinck et al., 2013), which is a bagging decision-tree based model (Ho, 1995), and "XGBoost" (XGB) and its corresponding python package (Chen and Guestrin, 2016). XGB is also a decision tree-based model, though it comes from the "boosting" family (Zhou, 2012). They were chosen because of the virtues of an ensemble learning algorithm, along with their usual good fit with temporal data. Additionally, we applied an artificial neural network (ANN), using the keras python package (Chollet et al., 2015). Lastly, we used simple Linear Regression (LR), also from the sklearn python package, as a baseline for comparison. The hyper-parameters of these models were found using a grid search. The best performance was exhibited by the XGBoost classifier with a step size of 0.05 . For the optimal feature group, the number of boosting rounds was 300 . All other parameters were run with the default settings. All results shown were cross-validated by dividing the data randomly to training and validation sets $(80 \%$ of the data were used for training, $20 \%$ of the data were used for validation) and repeating the process 10-20 times (determined by the time complexity of the analysis). Our main measure of model performance was $r^{2}$, which is simply the Pearson correlation squared. It is commonly interpreted as the proportion of the variance for a dependent variable that is explained by an independent variable or variables.

At first, we compared the different feature types in the prediction process with the different classifiers. Table 1

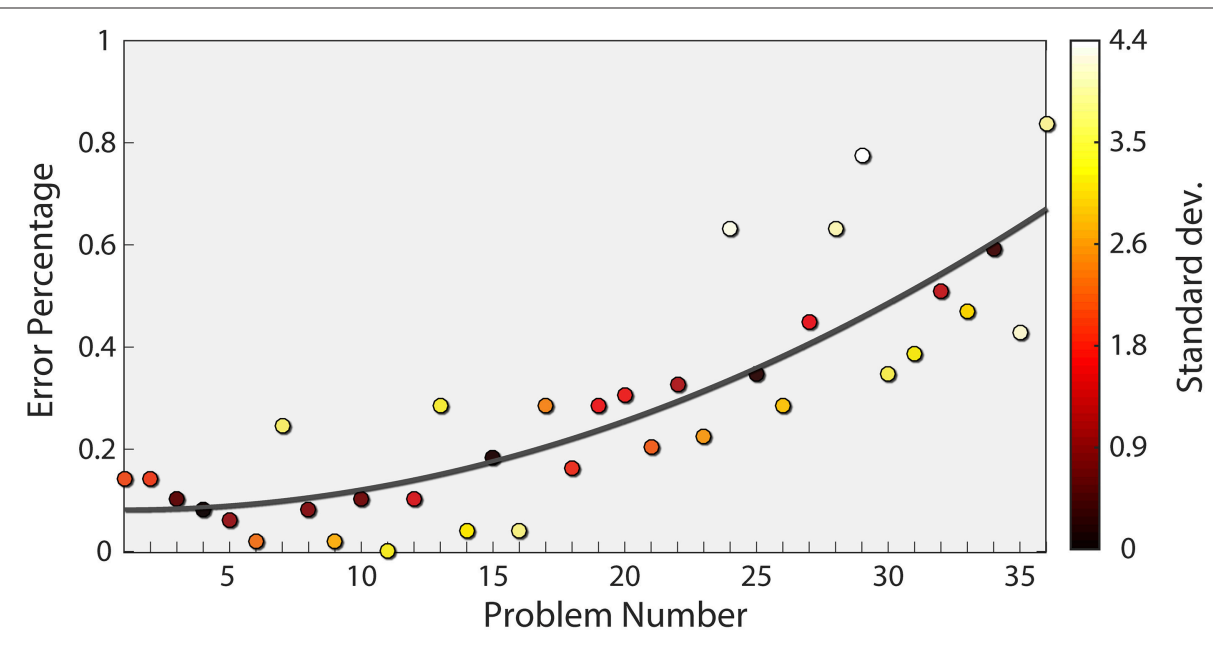

FIGURE 2 | Subject Error rate as a function of problem number. The mean error rate across subjects is plotted for each problem (circles) together with a quadratic fit (dark gray curve). The equation corresponding to the fit is $y=0.0004793 x^{2}-0.000897 x-0.08256$. The color of each point indicates the number of standard deviations from the fit, with bright colors indicating a higher value. 
TABLE 1 | The table shows the Pearson correlation $\left(r^{2}\right)$ of each Feature groupModel Pair.

\begin{tabular}{lcccc}
\hline & LR & RF & XGB & ANN \\
\hline PS & 0.007 & $0.383^{\star}$ & $0.655^{\star}$ & $0.346^{\star}$ \\
Complexity & $0.323^{\star}$ & 0.055 & $0.508^{\star}$ & $0.286^{\star}$ \\
Connectivity & $0.335^{\star}$ & 0.186 & $0.5^{\star}$ & $0.267^{*}$ \\
PS \& Complexity & 0 & $0.322^{\star}$ & $0.641^{\star}$ & $0.186^{\star}$ \\
PS \& Connectivity & 0.07 & $0.44^{*}$ & $0.67^{\star}$ & $0.32^{*}$ \\
Complexity \& Connectivity & $0.339^{\star}$ & 0.122 & $0.519^{\star}$ & $0.331^{\star}$ \\
All Features & 0.05 & $0.358^{\star}$ & $0.628^{\star}$ & $0.297^{\star}$ \\
\hline
\end{tabular}

summarizes the $r^{2}$ results for this analysis (all results marked with an ${ }^{\star}$ were significant). The best results were obtained using XGB for all feature types as seen in a variance $\operatorname{test}(F(36,3)=$ $16.79, p<0.001$, Tukey multiple comparisons: $p<0.05$ for all XGB pairs). XGB provides a good trade-off between model complexity and the number of samples required to reach robustness. Even though ANN can capture very complex relationships, they require a large training set. On the other hand, LR and RF do not require significant amounts of training data, but their model complexity is significantly more constrained than XGB.

Next, we compared the utility of each of the three different feature types. PS and connectivity features obtained the highest score, and adding the complexity features to either of the two did not contribute significantly to the prediction. This suggests that complexity features do not add any further information beyond spectral features and connectivity features. To test whether this was not due to high model complexity resulting in over-fitting, we conducted a feature selection process. We found that even after reducing the number of features, no combination of complexity, connectivity and PS features yielded better results than using only the PS and connectivity features together with the basic features.

Figure 3 shows a scatter plot of the best model's prediction together with the true label of each instance in the test set. The Pearson correlation of the best model is $r^{2}=0.67(p<0.01)$. The model was trained on the problem serial number, which should, in principle, produce a linear relationship. However, as evident in Figure 2, the relationship between problem number and error rate is slightly non-linear. This suggests that the relationship between problem number and the EEG measure could also be non-linear. We therefore also computed the Spearman correlation, which relates to a general monotonic relationship rather than a linear one, and obtained a value of 0.81 $(p<0.01)$. One of the features used by the algorithm was the duration of each segment, namely the time it took the subject to answer. We also examined the performance with only this feature and found a $r^{2}$ of $0.23(p<0.01)$ and a Spearman correlation of $0.41(p<0.01)$.

\subsection{Effect of Number of Electrodes}

From an applicative point of view, the number of electrodes affects both the cost and the complexity of using EEG. We therefore examined the extent to which reducing the number of electrodes affects the prediction quality. To this end, we

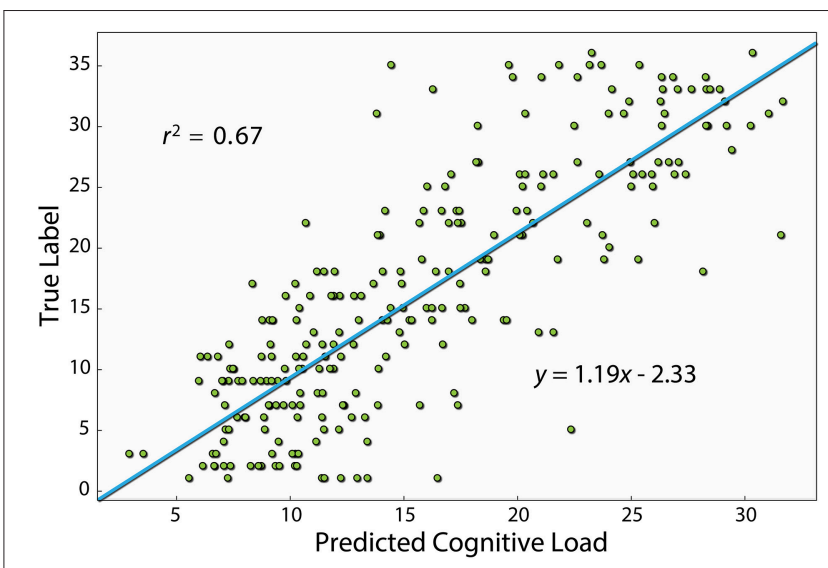

FIGURE 3 | This figure shows the Pearson correlation between the XGBoost model's prediction and the true label of each instance. The model shown here uses the PS, connectivity and basic features, which is the one that produced the best prediction. The equation of the linear fit is $y=1.19 x-2.33$.

conducted a two step analysis. Firstly, we ran 1,000 simulations, where in each, ten electrodes were chosen randomly out of the total of 62 . For each electrode combination, only the relevant PS features were used (five per channel, in addition to the basic features) to generate a workload prediction using the XGB algorithm. We then sorted the electrodes based on the percentage of simulations each electrode was involved in that yielded a score above a specified threshold, out of all simulations it participated in. The top thirty electrodes were chosen in descending order and were taken for the second step, where the effect of the number of best electrodes on the $r^{2}$ was examined. As seen in Figure 4, a relatively high $r^{2}$ of 0.7 ( $\left.p<0.01\right)$ can be obtained using only 12 electrodes (and in fact over 95 percent of peak performance for only 8). Additionally, using the same features of the 12 electrodes, the model produces a Spearman correlation of $0.82(p<0.05)$. These 12 electrodes were: CP1, CPz, CP4, TP8, TP10, P3, P4, PO7, O1, O2, AF3, FT8.

\subsection{Effect of Discretizing the Workload}

In our analysis, the target variable (difficulty of each problem) had 34 possible values. We analyzed the influence of reducing the number of levels of the target variable. We used different sized bins, to reduce the number of different values to $6,9,18,34$. For example, to obtain 6 levels, values were binned to [1-6], [7-12], [13-18], [19-24], [25-30], and [31-36]. As evident in Figure 5, prediction quality generally decreased with the number of levels. This is not surprising, because the prediction task becomes more complex with the number of levels. In addition, we show that using only the best 12 electrodes found earlier to compute the connectivity features (combined with the PS features of those electrodes), we obtain $r^{2}=0.713(p<0.05)$ for 6 levels, which is the best prediction quality we obtained.

\subsection{Individualized Prediction Using Neural Networks}

Lastly, because different individuals might experience different levels of cognitive load for the same problem, we wanted to assess 


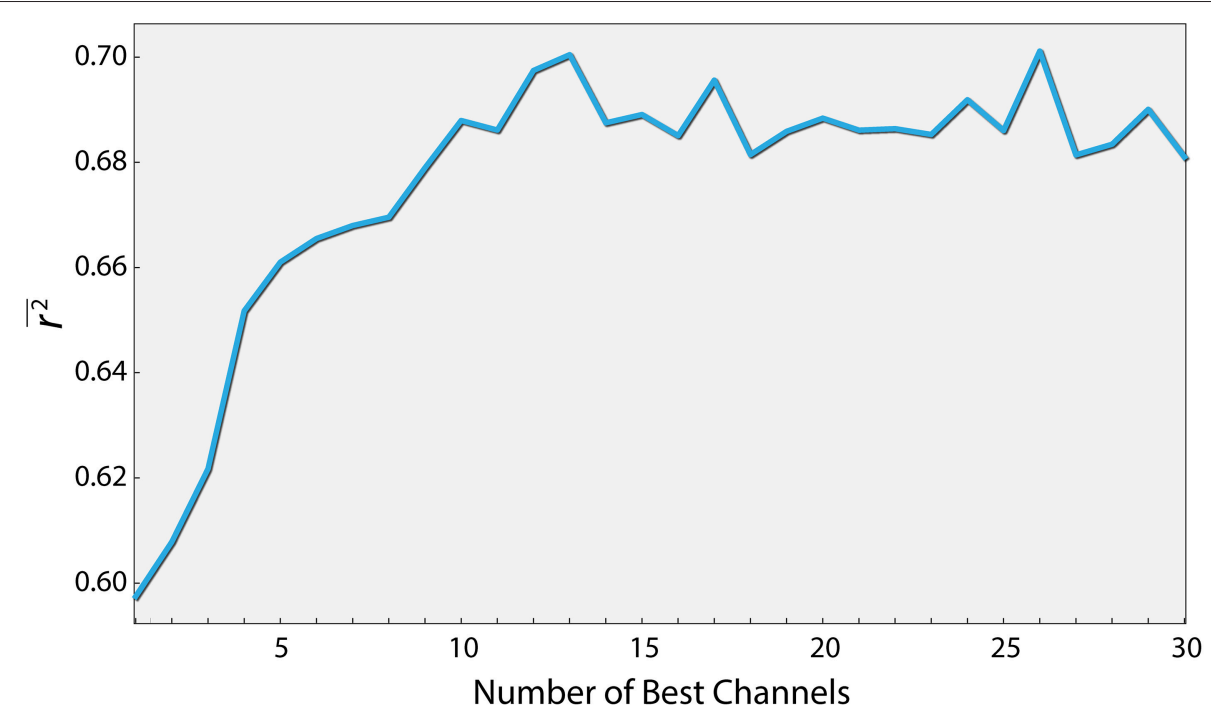

FIGURE 4 | Performance as a function of the number of best channels. Channels were ordered according to their contribution to the prediction quality (see text for details). The curve depicts the prediction quality $\left(r^{2}\right)$ for the XGBoost algorithm as a function of the number of best channels taken into account.

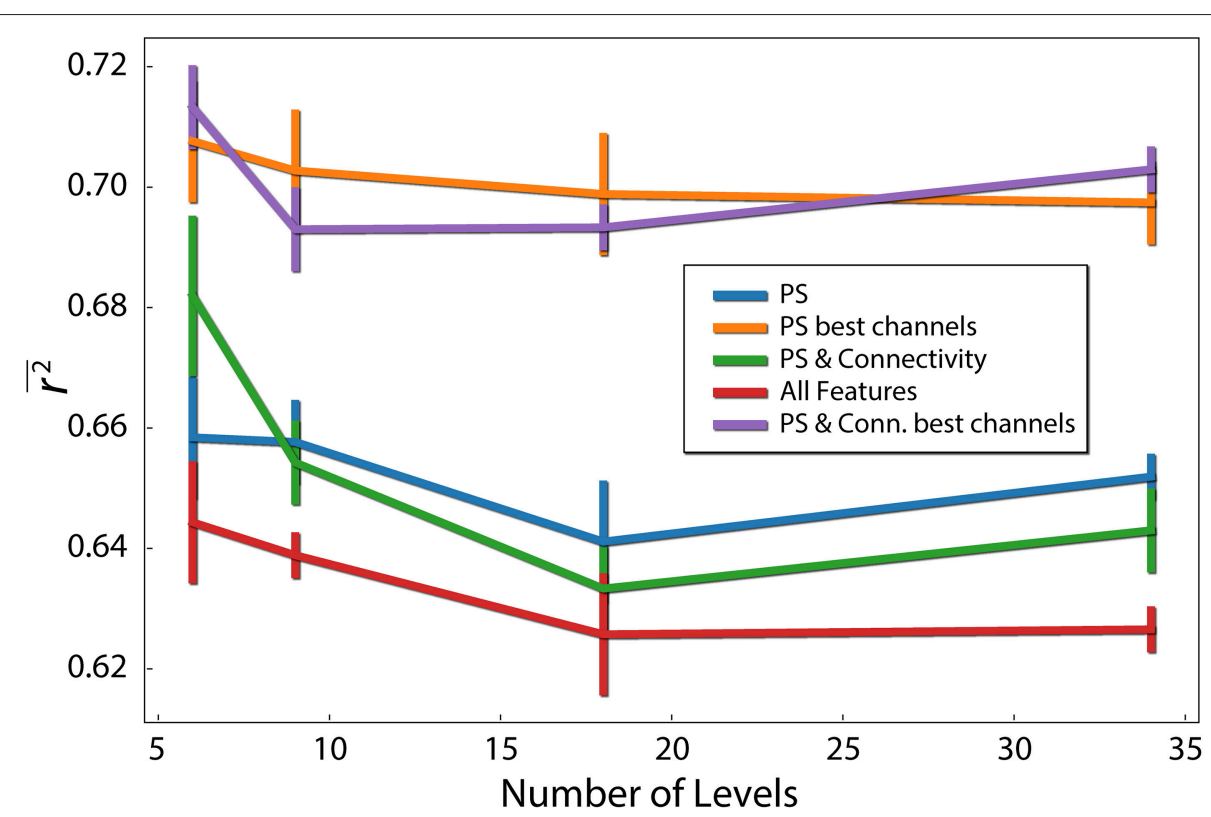

FIGURE 5 | Difficulty level discretization effect on prediction quality $\left(r^{2}\right)$. Each line corresponds to different feature types. PS red are the PS features of the 12 best channels. Error bars reflect standard error of the mean.

the influence of individualizing the prediction model. To this end, we first built a three layer artificial neural network (ANN), trained with data from all subjects using the PS and connectivity features of the 12 best electrodes. We then fixed the parameters of the first and second layers, and for each subject continued to train the weights of the output layer (Figure 6). This is a common practice in the field of neural networks (Gruber et al., 2017). We conducted a paired t-test (Figure 7), by calculating the mean correlation with the correct answer over several folds using the general model $(M=0.39, S D=0.06)$ and after tuning $(M=0.43, S D=0.06)$, which yielded a significant difference $(t=-4.75, p=0.001)$ in favor of the individualized network models.

\section{DISCUSSION}

We recorded EEG from subjects while solving the advanced progressive matrices test (Raven's matrices test) and used EEG features and machine learning to predict problem difficulty, our chosen operationalization of cognitive workload. Problem 


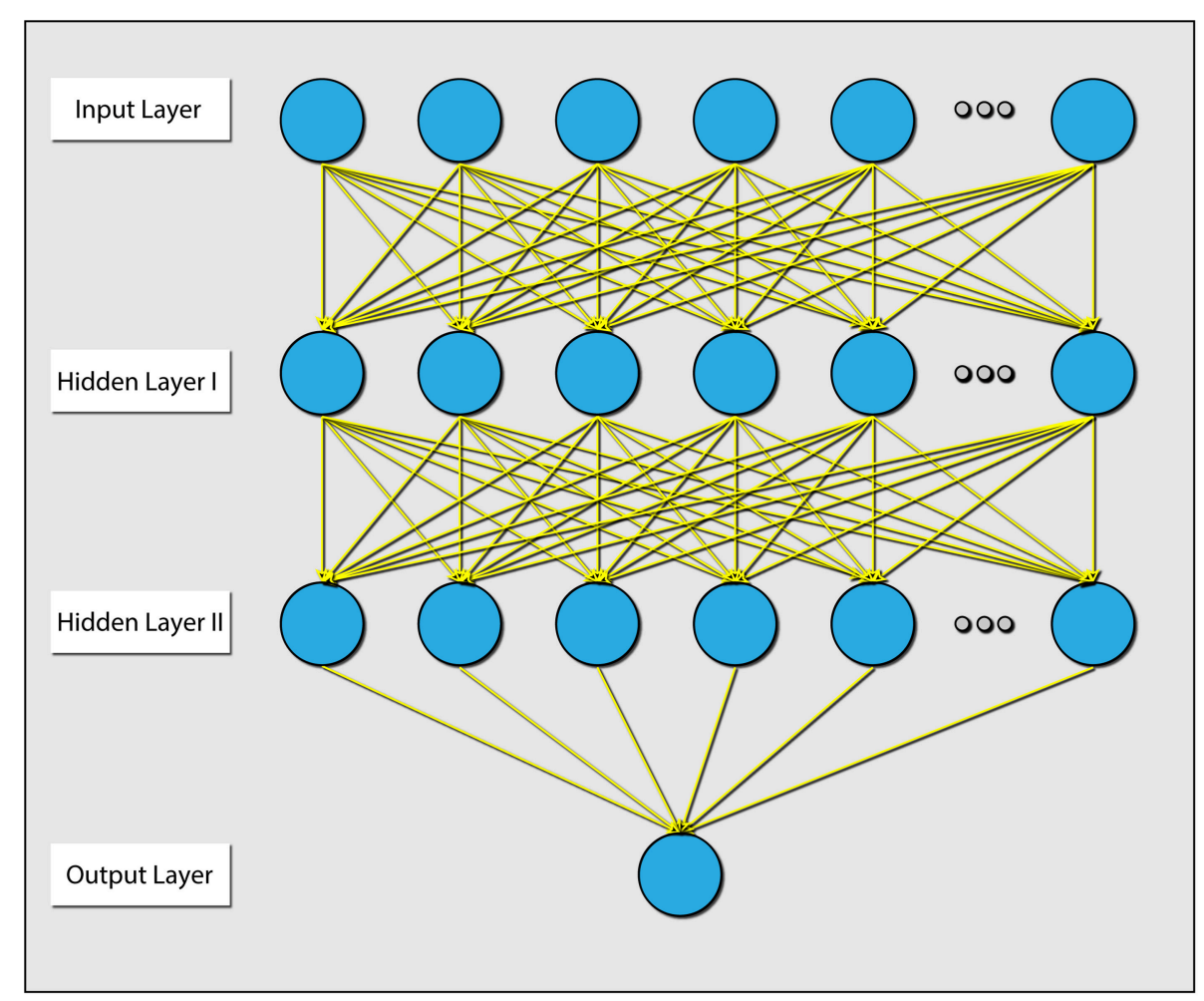

FIGURE 6 | Diagram explaining the architecture of the ANN that was used. There were 2 hidden layers, and all layers were dense (e.g., all connections were present). The parameters between the input layer and hidden layer 1 , and the parameters between hidden layer 1 and hidden layer 2 were held during the individualization phase.

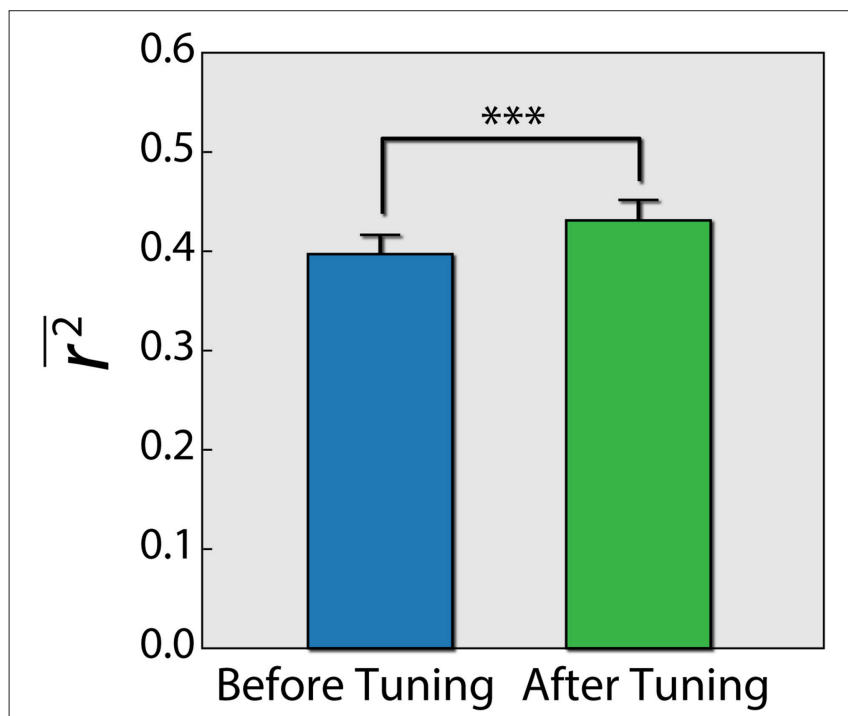

FIGURE 7 | Difference of $r^{2}$ score using an ANN before and after individualizing the last layer for each subject. Error bars reflect standard error of the mean. Paired $t$-test results are shown. ${ }^{* \star} p=0.001$.

difficulty was ordered on a scale from 1 to 36 (Forbes, 1964; Arthur et al., 1999) and was treated here as a continuous value. Our results show that even when considering cognitive load in a continuous manner, a reasonable prediction accuracy can be obtained using EEG measures. This could be very useful for many applications in which there is a wide range of cognitive workload levels. These findings extend those of previous studies which used a small number (2-3) of discrete levels of cognitive workload (Gerě and Jaušcvec, 1999; McDonald and Soussou, 2011; Conrad and Bliemel, 2016). Indeed, we found that reducing the number of difficulty levels improves the results significantly.

We examined several machine learning algorithms and found that XGBoost outperformed all other algorithms with all three feature groups. XGBoost was more accurate than the simpler models of linear regression and Random Forest. The lower scores of the ANN are probably due to the fact that they typically require a much larger training dataset than we had at our disposal (Chen and Guestrin, 2016). Furthermore, even though the ANN scored lower than XGBoost, we showed that prediction quality can be improved by tuning the last layer of the ANN to each individual. With a larger dataset, the personalized ANN could potentially attain better prediction than XGBoost. Additionally, in this study we did not use individual features such as individual frequency bands. In general, this could improve the performance of the algorithm. Incorporating individual features should be addressed in future research.

As part of our analysis, we checked the impact of additional EEG measures, specifically metrics of connectivity and metrics of neural complexity. Our results suggest that connectivity measures do add information regarding cognitive load beyond the simple spectral features. On the other hand, it seems 
that complexity features, while holding information regarding cognitive load, do not afford additional information over and above that found in connectivity and PS features.

Lastly, we found that prediction quality did not deteriorate, and even improved, when using a limited number of channels ( 12), which is important for practical applications. This is most probably due to better generalization, resulting from a less complex model, as opposed to one utilizing all channels.

We chose to utilize the advanced progressive matrices test in this study because of the high validity of its operationalization of difficulty levels. However, to extend our findings further toward applicability, future studies should examine the utility of our EEG-based metrics for cognitive load in real-life settings such as control tower operator performance as aerial traffic ebbs and flows. Since our results indicate the feasibility of employing an array comprising as little as eight electrodes, potentially such studies could be carried out in parallel using portable dry EEG systems. The added benefit would be the feasibility of amassing the expansive datasets necessary for utilizing elaborate neural network models, which in this scenario are expected to improve predictive ability. In addition, it would be useful to identify EEG markers for different dimensions of cognitive workload. Such markers would pave the way for optimizing and personalizing learning processes from e-learning to military training (Ikehara and Crosby, 2005; Mills et al., 2017).

\section{REFERENCES}

Abásolo, D., Hornero, R., Gómez, C., García, M., and López, M. (2006). Analysis of eeg background activity in Alzheimer's disease patients with lempel-ziv complexity and central tendency measure. Med. Eng. Phys. 28, 315-322. doi: 10.1016/j.medengphy.2005.07.004

Aricò, P., Borghini, G., Di Flumeri, G., Colosimo, A., Bonelli, S., Golfetti, A., et al. (2016a). Adaptive automation triggered by eeg-based mental workload index: a passive brain-computer interface application in realistic air traffic control environment. Front. Hum. Neurosci. 10:539. doi: 10.3389/fnhum.201 6.00539

Aricò, P., Borghini, G., Di Flumeri, G., Colosimo, A., Pozzi, S., and Babiloni, F. (2016b). A passive brain-computer interface application for the mental workload assessment on professional air traffic controllers during realistic air traffic control tasks. Prog. Brain Res. 228, 295-328. doi: 10.1016/bs.pbr.2016.04.021

Arthur, W. Jr., Tubre, T. C., Paul, D. S., and Sanchez-Ku, M. L. (1999). College-sample psychometric and normative data on a short form of the raven advanced progressive matrices test. J. Psychoeduc. Assess. 17, 354-361. doi: $10.1177 / 073428299901700405$

Bell, A. J., and Sejnowski, T. J. (1995). An information-maximization approach to blind separation and blind deconvolution. Neural Comput. 7, 1129-1159. doi: 10.1162/neco.1995.7.6.1129

Borghini, G., Aricò, P., Di Flumeri, G., Cartocci, G., Colosimo, A., Bonelli, S., et al. (2017). Eeg-based cognitive control behaviour assessment: an ecological study with professional air traffic controllers. Sci. Rep. 7, 547. doi: 10.1038/s41598-017-00633-7

Brainard, D. H., and Vision, S. (1997). The psychophysics toolbox. Spat. Vis. 10, 433-436. doi: 10.1163/156856897X00357

Brouwer, A.-M., Hogervorst, M. A., Van Erp, J. B., Heffelaar, T., Zimmerman, P. H., and Oostenveld, R. (2012). Estimating workload using eeg spectral power and erps in the n-back task. J. Neural Eng. 9:045008. doi: 10.1088/1741-2560/9/4/045008

Buitinck, L., Louppe, G., Blondel, M., Pedregosa, F., Mueller, A., Grisel, O., et al. (2013). "API design for machine learning software: experiences from the

\section{ETHICS STATEMENT}

The protocol was approved by the Ben-Gurion University ethics committee. All subjects gave written informed consent in accordance with the Declaration of Helsinki and received compensation for participating.

\section{AUTHOR CONTRIBUTIONS}

NF, KG, and OS: experiment planning and design. NF: data acquisition. NF and TF: data analysis. NF, TF, KG, and OS: writing the manuscript.

\section{FUNDING}

This research was supported in part by the Helmsley Charitable Trust through the Agricultural, Biological and Cognitive (ABC) Robotics Initiative and by the Marcus Endowment Fund both at Ben-Gurion University of the Negev.

\section{ACKNOWLEDGMENTS}

The authors thank Dr. Nir Getter for helpful discussions on the manuscript and Ms. Koral Regev for valuable help in running the experiments.

scikit-learn project," in ECML PKDD Workshop: Languages for Data Mining and Machine Learning (Prague), 108-122.

Bullmore, E., Barnes, A., Bassett, D. S., Fornito, A., Kitzbichler, M., Meunier, D., et al. (2009). Generic aspects of complexity in brain imaging data and other biological systems. Neuroimage 47, 1125-1134. doi: 10.1016/j.neuroimage.2009.05.032

Bullmore, E., and Sporns, O. (2009). Complex brain networks: graph theoretical analysis of structural and functional systems. Nat. Rev. Neurosci. 10, 186. doi: $10.1038 / \mathrm{nrn} 2575$

Chen, T., and Guestrin, C. (2016). "Xgboost: a scalable tree boosting system," in Proceedings of the 22nd ACM Sigkdd International Conference on Knowledge Discovery and Data Mining (San Francisco, CA: ACM), 785-794. doi: $10.1145 / 2939672.2939785$

Chollet, F. et al. (2015). Keras. Available online at: https://keras.io. (accessed May, 2019).

Conrad, C. D., and Bliemel, M. (2016). "Psychophysiological measures of cognitive absorption and cognitive load in E-learning applications," in Proceedings of the 37th International Conference on Information Systems, December 11-14, eds P. Agerfalk, N. Levina, and S. S. Kien (Dublin).

Coyne, J. T., Baldwin, C., Cole, A., Sibley, C., and Roberts, D. M. (2009). "Applying real time physiological measures of cognitive load to improve training," in International Conference on Foundations of Augmented Cognition (San Diego, CA: Springer), 469-478.

Delorme, A., and Makeig, S. (2004). Eeglab: an open source toolbox for analysis of single-trial eeg dynamics including independent component analysis. $J$. Neurosci. Methods 134, 9-21. doi: 10.1016/j.jneumeth.2003.10.009

Fekete, T., Beacher, F. D., Cha, J., Rubin, D., and Mujica-Parodi, L. R. (2014). Small-world network properties in prefrontal cortex correlate with predictors of psychopathology risk in young children: a nirs study. NeuroImage 85, 345-353. doi: 10.1016/j.neuroimage.2013.07.022

Forbes, A. (1964). An item analysis of the advanced matrices. Br. J. Educ. Psychol. 34, 223-236. doi: 10.1111/j.2044-8279.1964.tb00632.x

Gerě, I., and Jaušcvec, N. (1999). Multimedia: Differences in cognitive processes observed with eeg. Educ. Technol. Res. Dev. 47, 5-14. doi: 10.1007/BF022 99630 
Gruber, I., Hlaváč, M., Železnỳ, M., and Karpov, A. (2017). "Facing face recognition with resnet: round one," in International Conference on Interactive Collaborative Robotics (Hatfield: Springer), 67-74.

Ho, T. K. (1995). "Random decision forests," in Proceedings of $3 \mathrm{rd}$ International Conference on Document Analysis and Recognition, Vol. 1, (Montreal, QC: IEEE), 278-282.

Ikehara, C. S., and Crosby, M. E. (2005). "Assessing cognitive load with physiological sensors," in Proceedings of the 38th Annual Hawaii International Conference on System Sciences, 295-303.

Kleiner, M., Brainard, D., Pelli, D., Ingling, A., Murray, R., Broussard, C., et al. (2007). What's new in psychtoolbox-3. Perception 36:1.

Logan, G. D. (1985). Skill and automaticity: Relations, implications, and future directions. Can. J. Psychol./Revue Can. Psychol. 39:367. doi: 10.1037/h0080066

Mak, J. N., Chan, R. H., and Wong, S. W. (2013). "Spectral modulation of frontal eeg activities during motor skill acquisition: task familiarity monitoring using single-channel eeg," in Engineering in Medicine and Biology Society (embc), 2013 35th Annual International Conference of the IEEE (Osaka: IEEE), 5638-5641.

McDonald, N. J., and Soussou, W. (2011). "Quasar's qstates cognitive gauge performance in the cognitive state assessment competition 2011," in Engineering in Medicine and Biology Society, EMBC, 2011 Annual International Conference of the IEEE (Boston, MA: IEEE), 6542-6546.

Mills, C., Fridman, I., Soussou, W., Waghray, D., Olney, A. M., and D’Mello, S. K. (2017). "Put your thinking cap on: detecting cognitive load using eeg during learning," in Proceedings of the Seventh International Learning Analytics \& Knowledge Conference (Vancouver, BC: ACM), 80-89.

Mullen, T. R., Kothe, C. A., Chi, Y. M., Ojeda, A., Kerth, T., Makeig, S., et al. (2015). Real-time neuroimaging and cognitive monitoring using wearable dry eeg. IEEE Trans. Biomed. Eng. 62, 2553-2567. doi: 10.1109/TBME.2015.2481482

Palinko, O., Kun, A. L., Shyrokov, A., and Heeman, P. (2010). "Estimating cognitive load using remote eye tracking in a driving simulator,' in Proceedings of the 2010 Symposium on Eye-Tracking Research \& Applications (Austin, TX: ACM), 141-144.

Pelli, D. G. (1997). The videotoolbox software for visual psychophysics: transforming numbers into movies. Spat. Vis. 10, 437-442. doi: 10.1163/156856897X00366

Peng, C.-K., Havlin, S., Stanley, H. E., and Goldberger, A. L. (1995). Quantification of scaling exponents and crossover phenomena in nonstationary heartbeat time series. Chaos 5, 82-87. doi: 10.1063/1.166141
Pope, A. T., Bogart, E. H., and Bartolome, D. S. (1995). Biocybernetic system evaluates indices of operator engagement in automated task. Biol. Psychol. 40, 187-195. doi: 10.1016/0301-0511(95) 05116-3

Raven, J. (2000). The raven's progressive matrices: change and stability over culture and time. Cogn. Psychol. 41, 1-48. doi: 10.1006/cogp.1999. 0735

Raven, J. C., and Court, J. H. (1998). Raven's Progressive Matrices and Vocabulary Scales. London: Psychological Corporation.

Rubin, D., Fekete, T., and Mujica-Parodi, L. R. (2013). Optimizing complexity measures for fmri data: algorithm, artifact, and sensitivity. PLoS ONE 8:e63448. doi: 10.1371/journal.pone.0063448

Stevens, R., Galloway, T., and Berka, C. (2006). "Integrating EEG models of cognitive load with machine learning models of scientific problem solving," in Augmented Cognition: Past, Present and Future, eds D. Schmorrow, K. Stanney, and L. Reeves (Arlington, VA: Strategic Analysis), $55-65$.

Sweller, J., Van Merrienboer, J. J., and Paas, F. G. (1998). Cognitive architecture and instructional design. Educ. Psychol. Rev. 10, 251-296. doi: 10.1023/A:1022193728205

Tononi, G., and Edelman, G. M. (1998). Consciousness and complexity. science 282, 1846-1851. doi: 10.1126/science.282.5395.1846

Zhang, X.-S., Roy, R. J., and Jensen, E. W. (2001). Eeg complexity as a measure of depth of anesthesia for patients. IEEE Trans. Biomed. Eng. 48, 1424-1433. doi: $10.1109 / 10.966601$

Zhou, Z.-H. (2012). Ensemble Methods: Foundations and Algorithms. Raton, FL: Chapman and Hall/CRC. doi: 10.1201/b12207

Conflict of Interest Statement: The authors declare that the research was conducted in the absence of any commercial or financial relationships that could be construed as a potential conflict of interest.

Copyright (c) 2019 Friedman, Fekete, Gal and Shriki. This is an open-access article distributed under the terms of the Creative Commons Attribution License (CC BY). The use, distribution or reproduction in other forums is permitted, provided the original author(s) and the copyright owner(s) are credited and that the original publication in this journal is cited, in accordance with accepted academic practice. No use, distribution or reproduction is permitted which does not comply with these terms. 